\title{
Soft phonons and ultralow lattice thermal conductivity in the Dirac semimetal $\mathrm{Cd}_{3} \mathrm{As}_{2}$
}

\author{
Shengying Yue $\odot,{ }^{1}$ Hamid T. Chorsi $\odot,{ }^{2}$ Manik Goyal,,${ }^{3}$ Timo Schumann, ${ }^{3}$ Runqing Yang, ${ }^{1}$ Tashi Xu, ${ }^{1}$ \\ Bowen Deng, ${ }^{1}$ Susanne Stemmer, ${ }^{3}$ Jon A. Schuller, ${ }^{2}$ and Bolin Liao $\oplus^{1, *}$ \\ ${ }^{1}$ Department of Mechanical Engineering, University of California, Santa Barbara, Santa Barbara, California 93106, USA \\ ${ }^{2}$ Department of Electrical and Computer Engineering, University of California, Santa Barbara, Santa Barbara, California 93106, USA \\ ${ }^{3}$ Materials Department, University of California, Santa Barbara, Santa Barbara, California 93106, USA
}

(Received 12 August 2019; published 14 November 2019)

\begin{abstract}
Recently, $\mathrm{Cd}_{3} \mathrm{As}_{2}$ has attracted intensive research interest as an archetypical Dirac semimetal, hosting threedimensional linear-dispersive electronic bands near the Fermi level. Previous studies have shown that singlecrystalline $\mathrm{Cd}_{3} \mathrm{As}_{2}$ has an anomalously low lattice thermal conductivity, ranging from 0.3 to $0.7 \mathrm{~W} / \mathrm{mK}$ at $300 \mathrm{~K}$, which has been attributed to point defects. In this work, we combine first-principles lattice dynamics calculations and temperature-dependent high-resolution Raman spectroscopy of high-quality single-crystal thin films grown by molecular-beam epitaxy to reveal the existence of a group of soft optical phonon modes at the Brillouin-zone center of $\mathrm{Cd}_{3} \mathrm{As}_{2}$. These soft phonon modes significantly increase the scattering phase space of heat-carrying acoustic phonons and are the origin of the low lattice thermal conductivity of $\mathrm{Cd}_{3} \mathrm{As}_{2}$. Furthermore, we show that the interplay between the phonon-phonon Umklapp scattering rates and the soft optical phonon frequency explains the unusual nonmonotonic temperature dependence of the lattice thermal conductivity of $\mathrm{Cd}_{3} \mathrm{As}_{2}$. Our results further suggest that the soft phonon modes are potentially induced by a Kohn anomaly associated with the Dirac nodes, in analogy to similar, nonetheless weaker, effects in graphene and Weyl semimetals.
\end{abstract}

DOI: 10.1103/PhysRevResearch.1.033101

\section{INTRODUCTION}

Cadmium arsenide $\left(\mathrm{Cd}_{3} \mathrm{As}_{2}\right)$ is a well-studied electronic material due to its semimetallicity and ultrahigh charge carrier mobility [1,2]. The research interest in $\mathrm{Cd}_{3} \mathrm{As}_{2}$ has been revived recently due to both theoretical prediction [3] and experimental observations [4-7] that $\mathrm{Cd}_{3} \mathrm{As}_{2}$ hosts threedimensional linear Dirac bands [8]. Namely, the electronic structure of $\mathrm{Cd}_{3} \mathrm{As}_{2}$ is a three-dimensional analog to that of graphene. Extensive optical and electrical transport measurements have been conducted on $\mathrm{Cd}_{3} \mathrm{As}_{2}$ to probe the Dirac physics of its electronic states [9-12]. In comparison, less-studied aspects of $\mathrm{Cd}_{3} \mathrm{As}_{2}$ are its lattice dynamics and heat transport properties, which are essential for thermal management of actual electronic devices utilizing this material. Furthermore, combining the recently predicted large thermopower of topological semimetals [13] and the ultrahigh charge carrier mobility, $\mathrm{Cd}_{3} \mathrm{As}_{2}$ is also a promising candidate for thermoelectric energy conversion applications [14-16], where a low thermal conductivity is required for high efficiency. Therefore, a thorough understanding of the lattice dynamics and thermal transport properties of $\mathrm{Cd}_{3} \mathrm{As}_{2}$ is of paramount importance for its practical applications. Moreover, how the lattice degree of freedom couples to topological

\footnotetext{
*bliao@ucsb.edu

Published by the American Physical Society under the terms of the Creative Commons Attribution 4.0 International license. Further distribution of this work must maintain attribution to the author(s) and the published article's title, journal citation, and DOI.
}

properties of the electronic structure is of fundamental interest and remains largely unexplored [17-19].

The thermal conductivity of crystalline $\mathrm{Cd}_{3} \mathrm{As}_{2}$ was first measured by Spitzer et al. [20] and Armitage and Goldsmid [21]. Subtracting the electronic contribution from the total thermal conductivity using the Wiedemann-Franz law, researchers found that $\mathrm{Cd}_{3} \mathrm{As}_{2}$ possessed an anomalously low lattice thermal conductivity $(0.3 \mathrm{~W} / \mathrm{mK}$ at $300 \mathrm{~K}$ reported by Spitzer et al. [20]). This value is lower than that of amorphous glass and many polymers and in sharp contrast to other crystalline materials with similar atomic mass and crystal structure. A recent study used a strong magnetic field up to $14 \mathrm{~T}$ to freeze out electron transport and measured a lattice thermal conductivity of around $0.7 \mathrm{~W} / \mathrm{mK}$ at $300 \mathrm{~K}$ [14]. More surprisingly, they found that the lattice thermal conductivity of $\mathrm{Cd}_{3} \mathrm{As}_{2}$ increases with temperature above roughly $300 \mathrm{~K}$, which runs counter to the familiar $1 / T$ dependence of lattice thermal conductivity in crystalline materials due to phonon-phonon Umklapp scatterings. So far, the origin of the anomalously low lattice thermal conductivity of $\mathrm{Cd}_{3} \mathrm{As}_{2}$ and its unusual temperature dependence remains unclear. Recent measurements of another Dirac semimetal, $\mathrm{ZrTe}_{5}$, also revealed highly anisotropic and very low thermal conductivity [22].

In this work, we combine first-principles lattice dynamics calculations and temperature-dependent high-resolution Raman measurement of high-quality $\mathrm{Cd}_{3} \mathrm{As}_{2}$ thin films grown by molecular beam epitaxy (MBE) to reveal the existence of a group of soft optical phonons in $\mathrm{Cd}_{3} \mathrm{As}_{2}$ with anomalously low frequencies at the Brillouin zone center. These soft optical phonons largely increase the scattering phase space of heat-carrying acoustic phonons and are responsible 
for the reduction of the lattice thermal conductivity. More interestingly, we find that the optical phonon frequency is highly sensitive to the smearing parameter of the electronic Fermi-Dirac distribution in our calculation. This is potentially a signature of the Kohn anomaly associated with the Dirac nodes, as has been found in graphene [23,24] and Weyl semimetal tantalum phosphide [25]. As a result, the frequency of the soft optical phonon increases with temperature, which reduces phonon-phonon scattering and leads to an increasing lattice thermal conductivity. Our investigation quantitatively reproduces the low lattice thermal conductivity of $\mathrm{Cd}_{3} \mathrm{As}_{2}$ and qualitatively explains its anomalous temperature dependence.

\section{RESULTS AND DISCUSSION}

\section{A. Electronic structure}

We used the Vienna ab initio simulation package (VASP) $[26,27]$ to calculate the electronic structure using density functional theory (DFT). The projector augmented wave [28,29] method was adopted with the Perdew-Burke-Ernzerhof [30] exchange correlation functional. The spin-orbit coupling was included in the calculation as implemented in VASP [31]. The cutoff energy for the plane-wave expansion was set to $500 \mathrm{eV}$. The Monkhorst-Pack $k$ mesh $6 \times 6 \times 6$ including the $\Gamma$ point was used to sample the whole Brillouin zone (BZ). The low-temperature phase of $\mathrm{Cd}_{3} \mathrm{As}_{2}$ below $748 \mathrm{~K}$ is body-centered tetragonal (bct) with the space group $\mathrm{I} 4_{1} / \mathrm{acd}$ and has a conventional cell consisting of 160 atoms [32] (the corresponding primitive cell includes 80 atoms), as shown in Fig. 1(a). The conventional cell can be constructed as a $2 \times 2 \times 4$ supercell of 10-atom substructures. Each substructure has an antifluorite-derived structure with arsenic atoms at the face-center locations and cadmium atoms that form a cube with two vacancies located diagonally on one surface of the cube [32], as illustrated in Fig. 1(b). The arrangement of the cadmium vacancies in the substructures alternates in a fashion depicted in Fig. 1(a), where the red arrows mark the positions of the cadmium vacancies. Before the electronic band structure calculations, the lattice structure was fully optimized with the Hellmann-Feynman force tolerance $0.001 \mathrm{eV} / \AA$. The lattice constants of the relaxed conventional cell are given in Fig. 1(a). The calculated electronic structure of $\mathrm{Cd}_{3} \mathrm{As}_{2}$ using the 80 -atom primitive cell is shown in Fig. 1(d), where the Dirac cones are located along the $\Gamma-Z$ direction at $\left(0,0, \pm 0.043 \AA^{-1}\right)$, in agreement with previous reports $[8,32,33]$. The $\mathrm{BZ}$ shape, high-symmetry points, and locations of the Dirac cones are shown in Fig. 1(c).

\section{B. Phonon dispersions}

Next we used first-principles calculation to examine the phonon dispersion relations of $\mathrm{Cd}_{3} \mathrm{As}_{2}$. For this calculation, we obtained the harmonic interatomic force constants (IFCs) by employing the finite displacement method based on DFT force calculations [34] implemented in VASP and then diagonalized the dynamical matrices to generate the phonon dispersion relations using PHONOPY [35]. To understand the effect of the short-range arrangement of cadmium vacancies, we calculated and compared the phonon dispersion relations of both the 80 -atom bct primitive cell and a crystal (a)
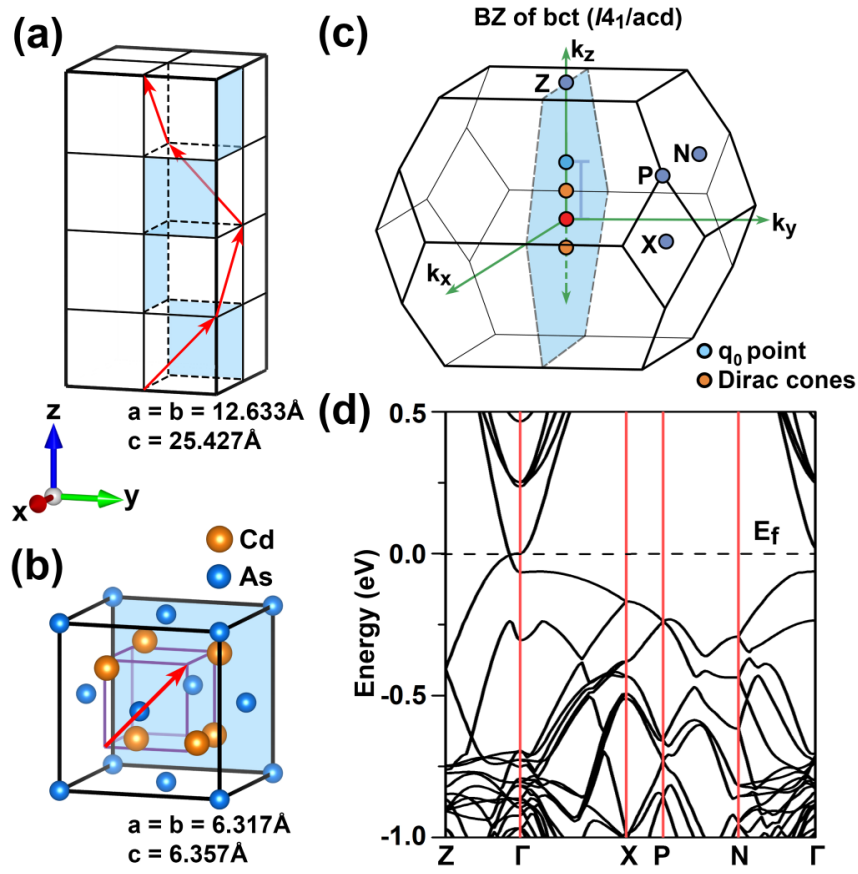

FIG. 1. Crystal structure and calculated electronic bands of $\mathrm{Cd}_{3} \mathrm{As}_{2}$. (a) Schematic of the $\mathrm{Cd}_{3} \mathrm{As}_{2}$ conventional cell. (b) Structure of the 10-atom building block for the full cell shown in (a). (c) First Brillouin zone of $\mathrm{Cd}_{3} \mathrm{As}_{2}$, where the location of the Dirac nodes and the position of the Kohn anomaly $\mathbf{q}_{0}$ are marked. (d) Calculated electronic band structure of $\mathrm{Cd}_{3} \mathrm{As}_{2}$.

constructed by the 10-atom substructure shown in Fig. 1(b) as its primitive cell. The calculated phonon dispersions along the $\Gamma-Z$ direction are presented in Fig. 2. The phonon dispersions along other high-symmetry lines in the BZ are given in [36]. We notice that the phonon dispersions calculated using the primitive cell and the substructure agree with each other very well near the zone center considering zone folding. This can be explained by the fact that the long-wavelength phonons near the zone center should be less sensitive to the short-range arrangement of the cadmium vacancies. A prominent feature of the phonon dispersions is the presence of a group of soft optical phonon modes at the zone center below $1 \mathrm{THz}$. In particular, the frequency of the lowest optical branch (LOB) is highly sensitive to the smearing parameter $\sigma$ of the electronic Fermi-Dirac distribution used in the DFT calculation. Here $\sigma$ (in units of $\mathrm{eV}$ ) represents the broadening of the electronic occupation around the Fermi level and a fictitious electronic temperature $T_{\mathrm{el}}=\sigma / k_{B}$ can be defined, where $k_{B}$ is the Boltzmann constant. $\operatorname{In~} \mathrm{Cd}_{3} \mathrm{As}_{2}$, we found that the frequency of the LOB decreases monotonically with decreasing $\sigma$, approaching roughly $0.1 \mathrm{THz}$ with $\sigma=0.01 \mathrm{eV}\left(T_{\mathrm{el}}=115 \mathrm{~K}\right)$. In comparison, the well-known high-performance thermoelectric materials with soft optical phonons, such as $\mathrm{Bi}_{2} \mathrm{Te}_{3}$ [37] and $\mathrm{PbTe}$ [38], have their LOBs around $1 \mathrm{THz}$.

This strong dependence of optical phonon frequencies on $\sigma$ was also observed in graphene, where optical phonons at the $\Gamma$ and $K$ points in the Brillouin zone showed similar behaviors [23]. In graphene, this phenomenon was attributed to the Kohn anomaly [39]. Kohn pointed out that, in a metal, the resonance between lattice vibrations and the Fermi surface can lead to 

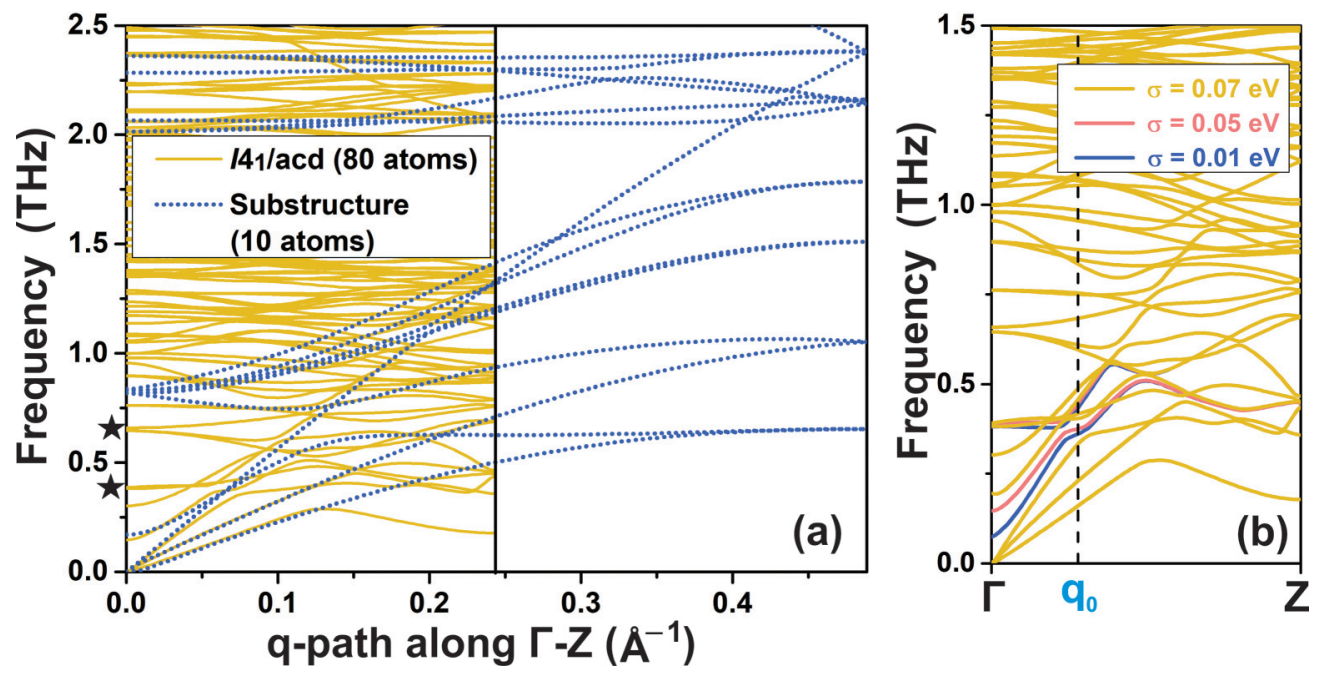

FIG. 2. (a) Calculated phonon dispersion relations of $\mathrm{Cd}_{3} \mathrm{As}_{2}$, using both the full 80 -atom primitive cell and the 10 -atom substructure. The stars mark the modes observed in the Raman measurement. (b) Phonon dispersion calculated using the full 80-atom primitive cell showing the dependence of the LOB frequency on the electronic smearing parameter $\sigma$. Here $\mathbf{q}_{0}$ marks the position of the potential Kohn anomaly in addition to $\Gamma$.

nonanalytic points in the phonon dispersion. These anomalies should happen when $\mathbf{q}=2 \mathbf{k}_{\mathrm{f}}$, where $\mathbf{q}$ is the phonon wave vector and $\mathbf{k}_{\mathrm{f}}$ is the Fermi wave vector [39]. Here $\mathbf{q}=2 \mathbf{k}_{\mathrm{f}}$ is the maximum wave vector of a phonon that can be involved in a scattering event with two electronic states on the Fermi surface. In graphene, the Fermi surface is virtually two Dirac points located at $K$ and $K^{\prime}$; therefore Kohn anomaly can only happen at $\Gamma$ (intranode scattering) and $K$ points (internode scattering between $K$ and $K^{\prime}$ ). In a Dirac material, the size of the Fermi surface is highly sensitive to the electronic smearing parameter $\sigma$, which explains the strong dependence of the optical phonon frequency in graphene on $\sigma$. In $\mathrm{Cd}_{3} \mathrm{As}_{2}$, the two Dirac nodes are located at $\left(0,0, \pm 0.043 \AA^{-1}\right)$. Therefore, Kohn anomalies should potentially appear at $\Gamma$ (intranode scattering) and $\mathbf{q}_{0}=\left(0,0, \pm 0.086 \AA^{-1}\right.$ ) (internode scattering), as marked in Figs. 1(c) and 2(b). In addition to the drastic phonon softening at $\Gamma$, a slight dip of one optical phonon branch at $\left(0,0, \pm 0.086 \AA^{-1}\right)$ is observed, again signaling the presence of Kohn anomaly. We emphasize here that the current calculation can only capture the Kohn anomaly due to static electronic screening of lattice vibrations, while a full treatment including dynamic screening effect will require the calculation of dynamic electron-phonon coupling that was done in the case of graphene [24] but is currently inaccessible for $\mathrm{Cd}_{3} \mathrm{As}_{2}$ given its complex structure.

\section{Raman measurements}

To verify the existence of the group of low-frequency optical phonons at $\Gamma$, we conducted temperature-dependent Raman measurement of high-quality $\mathrm{Cd}_{3} \mathrm{As}_{2}$ thin films grown by MBE [2]. The $\mathrm{Cd}_{3} \mathrm{As}_{2}$ layer is $\sim 30 \mathrm{~nm}$ thick and grown on a (111) $\mathrm{GaSb} / \mathrm{GaAs}$ substrate. Growth details and electrical characterization have been reported elsewhere [2]. We have recently estimated that the Fermi level is 70 and $80 \mathrm{meV}$ above the charge neutral point in $280-$ and $450-\mathrm{nm} \mathrm{Cd}_{3} \mathrm{As}_{2}$ thin films, respectively, grown with the same technique and conditions [40], as compared to $200 \mathrm{meV}$ typically seen in bulk samples [3,4]. We expect that the Fermi level of the 30-nm film we measured is even closer to the charge neutral point as we have observed strong signatures of Dirac physics in transport measurements in these films, as reported previously [41]. Therefore, we believe that the Raman measurements of the 30-nm thin film samples qualitatively correspond to our DFT calculations, where the Fermi level is placed at the charge neutral point. We used unpolarized excitation with a 488-nm wavelength and $65 \mathrm{~mW}$ power for the Raman measurement at 300,120 , and $77 \mathrm{~K}$. To rule out the influence of the substrate, we also measured the Raman spectrum of the substrate with the same configuration (provided in [36]). As compared to existing reports of Raman spectra of bulk $\mathrm{Cd}_{3} \mathrm{As}_{2}$ $[15,42-44]$, our measurement, as shown in Fig. 3, revealed the existence of a low-frequency optical phonon mode near $20 \mathrm{~cm}^{-1}$ at all three temperatures and another mode near $15 \mathrm{~cm}^{-1}$ at 120 and $77 \mathrm{~K}$. The spectral region with smaller Raman shift is masked by a strong background signal due to quasielastic electronic scatterings [43] and thus the LOB cannot be resolved. The frequencies of both resolved modes agree well with our first-principles calculation. In addition, we observed a decreasing trend of the frequency of the phonon mode near $20 \mathrm{~cm}^{-1}$ as the temperature was lowered, which was in contrast to the opposite temperature dependence of optical phonons with higher frequencies [43] caused by thermal expansion.

\section{Thermal conductivity}

Finally, we investigated how the existence of soft optical phonon modes affects the lattice thermal conductivity of $\mathrm{Cd}_{3} \mathrm{As}_{2}$. It is well understood that low-frequency optical phonon modes can significantly increase the available phase space for phonon-phonon scattering of heat-carrying acoustic phonons, which is responsible for the low lattice thermal 


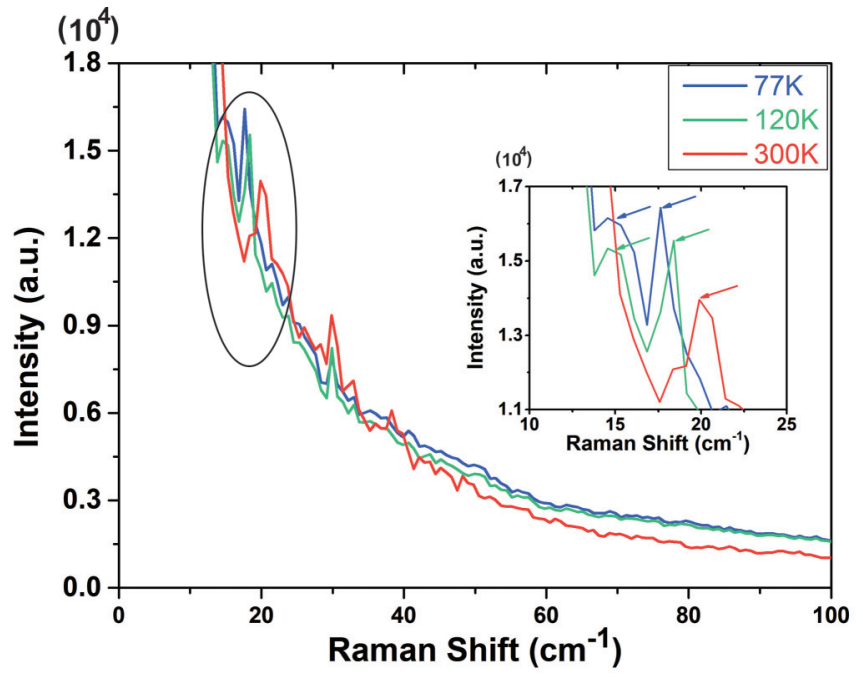

FIG. 3. Raman spectrum of $\mathrm{Cd}_{3} \mathrm{As}_{2}$ measured at three different temperatures. The inset shows the enlarged low-frequency region and the arrows mark the Raman peaks resolved by the measurement.

conductivity of the best thermoelectric materials, such as $\mathrm{Bi}_{2} \mathrm{Te}_{3}[37,45,46]$, PbTe [38,47], and SnSe [48]. In these materials, the soft optical phonon mode usually signals their proximity to a structural phase transition. Here we expect that the low-lying optical phonon modes in $\mathrm{Cd}_{3} \mathrm{As}_{2}$ will have a similar effect. Due to the complexity and low symmetry of the primitive cell of $\mathrm{Cd}_{3} \mathrm{As}_{2}$, it is computationally intractable to calculate the anharmonic force constants and phonon-phonon scattering rates using the full cell. To overcome this obstacle, we examined the thermal transport properties of the crystal constructed from the 10-atom substructures. The similarity between the dispersion relations of the long-wavelength phonons calculated using the full cell and the 10-atom substructure, as observed in Fig. 2(a), provides justification to this approach, since these long-wavelength phonons are major heat carriers in $\mathrm{Cd}_{3} \mathrm{As}_{2}$. In particular, we expect that this approach can qualitatively capture the physics of the soft phonon modes and their impact on thermal transport. Another unique aspect is the strong dependence of the LOB frequency on the electronic smearing parameter $\sigma$, which implies that the actual phonon dispersion of $\mathrm{Cd}_{3} \mathrm{As}_{2}$ will be highly sensitive to temperature. To include this effect, we analyzed thermal transport properties based on phonon dispersion relations assuming different values of $\sigma$. We calculated the lattice thermal conductivity $\kappa_{\text {ph }}$ by solving the phonon Boltzmann transport equation iteratively using ShengBTE [49]. The anharmonic third-order IFCs were calculated using the finite displacement method [50,51]. A $2 \times 2 \times 2$ supercell was used for the calculation and the interactions between atoms were taken into account up to sixth nearest neighbors. The convergence of $\kappa_{\mathrm{ph}}$ with the interaction distance between atoms was checked. The $q$-space (phonon momentum space) sampling grid was set to $12 \times 12 \times 12$. The grid density convergence of all cases was examined and all the $\kappa_{\mathrm{ph}}$ reported here are converged values.

Our results are shown in Fig. 4(a). Here the lattice temperature $T_{\mathrm{ph}}$ determines the Bose-Einstein distribution of the phonons involved in the phonon-phonon scattering calculation, while $\sigma$ dictates the broadening of the Fermi-Dirac distribution of electrons used in the DFT calculation. For a given $\sigma$, the lattice thermal conductivity decreases with an increasing lattice temperature, which is typical for crystalline materials limited by phonon-phonon Umklapp scattering. At $300 \mathrm{~K}$, our calculated lattice thermal conductivity is in the range of $0.3-0.9 \mathrm{~W} / \mathrm{mK}$, in good agreement with experimental reports $[14,20,21]$. For a given lattice temperature, the calculated lattice thermal conductivity is lower with a smaller $\sigma$. This is expected as a smaller $\sigma$ leads to a lower LOB frequency, which in turn causes stronger scattering of low-frequency acoustic phonons. We confirm this hypothesis by calculating the phonon-phonon scattering rates at a fixed lattice temperature but with two different $\sigma$ values, as shown in Fig. 4(b). While the scattering rates of higher-frequency optical phonons above $2 \mathrm{THz}$ are almost identical in the two cases, the scattering rates of low-frequency heat-carrying acoustic phonons are significantly enhanced with a smaller $\sigma$.

In order to make a direct connection to experimental results, both the phonon-phonon Umklapp scattering and the $\sigma$-dependent optical phonon frequency need to be taken into account. The counteraction of the two effects is expected to generate a nonmonotonic temperature dependence of the lattice thermal conductivity in $\mathrm{Cd}_{3} \mathrm{As}_{2}$. A rigorous treatment would entail a dynamic calculation [24,52] that is beyond the
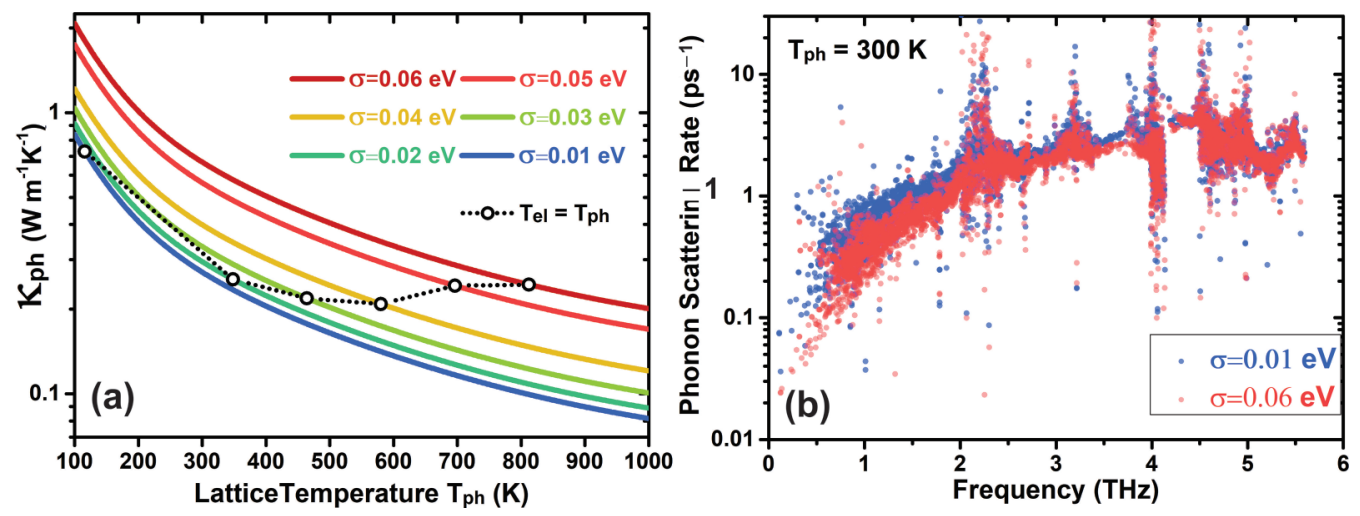

FIG. 4. (a) Calculated lattice thermal conductivity of $\mathrm{Cd}_{3} \mathrm{As}_{2}$ as a function of lattice temperature based on the phonon dispersion relations calculated using different $\sigma$ values. The open circles and dotted line mark the lattice thermal conductivity values obtained when $T_{\mathrm{el}}=T_{\mathrm{ph}}$. (b) Phonon-phonon scattering rates as a function of phonon frequency at $T_{\mathrm{ph}}=300 \mathrm{~K}$ with two different $\sigma$ values. 
current scope. However, to qualitatively capture the interplay of the two effects, we can compare the lattice thermal conductivity values calculated when the fictitious electronic temperature $T_{\mathrm{el}}$ determined by $\sigma$ is set equal to the lattice temperature $T_{\mathrm{ph}}$. These values are marked in Fig. 4(a) with open circles and connected by a dotted line. Below $450 \mathrm{~K}$, the temperature dependence of phonon-phonon Umklapp scattering is more prominent, and thus the lattice thermal conductivity decreases with temperature. Above $450 \mathrm{~K}$, however, the increasing optical phonon frequency plays a more important role and the lattice thermal conductivity starts to increase with temperature. This physical picture provides a qualitative explanation for the increasing trend of the lattice thermal conductivity of $\mathrm{Cd}_{3} \mathrm{As}_{2}$ with temperature as observed experimentally [14], while the quantitative difference from the experimental result might be due to the simplified treatment used here.

\section{CONCLUSION}

In summary, we investigated the lattice dynamics and thermal transport of Dirac semimetal $\mathrm{Cd}_{3} \mathrm{As}_{2}$ using firstprinciples calculation and Raman measurement. We identified the existence of soft optical phonons likely due to Kohn anomaly associated with the Dirac nodes. Based on the observation of the soft modes, we explained the ultralow lattice thermal conductivity of $\mathrm{Cd}_{3} \mathrm{As}_{2}$ due to soft-mode-enhanced phonon-phonon scatterings. We further suggested that the interplay of phonon-phonon Umklapp scattering and the optical phonon frequency can potentially explain the anomalous temperature dependence of the lattice thermal conductivity of $\mathrm{Cd}_{3} \mathrm{As}_{2}$ as observed experimentally. Our work exemplifies the rich phonon physics in topological materials.

\section{ACKNOWLEDGMENTS}

This work was based on research supported by the U.S. Department of Energy, Office of Basic Energy Sciences, Division of Materials Science and Engineering through the Early Career Research Program under Award No. DE-SC0019244. B.L. acknowledges support provided by the Regents' Junior Faculty Fellowship from the University of California, Santa Barbara. M.G., T.S., and S.S. acknowledge support through a Vannevar Bush Faculty Fellowship program by the U.S. Department of Defense (Grant No. N00014-16-1-2814).
[1] A. J. Rosenberg and T. C. Harman, $\mathrm{Cd}_{3} \mathrm{As}_{2}-\mathrm{A}$ noncubic semiconductor with unusually high electron mobility, J. Appl. Phys. 30, 1621 (1959).

[2] T. Schumann, M. Goyal, H. Kim, and S. Stemmer, Molecular beam epitaxy of $\mathrm{Cd}_{3} \mathrm{As}_{2}$ on a III-V substrate, APL Mater. 4, 126110 (2016).

[3] Z. Wang, H. Weng, Q. Wu, X. Dai, and Z. Fang, Threedimensional Dirac semimetal and quantum transport in $\mathrm{Cd}_{3} \mathrm{As}_{2}$, Phys. Rev. B 88, 125427 (2013).

[4] S. Borisenko, Q. Gibson, D. Evtushinsky, V. Zabolotnyy, B. Büchner, and R. J. Cava, Experimental Realization of a ThreeDimensional Dirac Semimetal, Phys. Rev. Lett. 113, 027603 (2014).

[5] Z. Liu, J. Jiang, B. Zhou, Z. Wang, Y. Zhang, H. Weng, D. Prabhakaran, S. Mo, H. Peng, P. Dudin et al., A stable threedimensional topological Dirac semimetal $\mathrm{Cd}_{3} \mathrm{As}_{2}$, Nat. Mater. 13, 677 (2014).

[6] S. Jeon, B. B. Zhou, A. Gyenis, B. E. Feldman, I. Kimchi, A. C. Potter, Q. D. Gibson, R. J. Cava, A. Vishwanath, and A. Yazdani, Landau quantization and quasiparticle interference in the three-dimensional Dirac semimetal $\mathrm{Cd}_{3} \mathrm{As}_{2}$, Nat. Mater. 13, 851 (2014).

[7] M. Neupane, S.-Y. Xu, R. Sankar, N. Alidoust, G. Bian, C. Liu, I. Belopolski, T.-R. Chang, H.-T. Jeng, H. Lin et al., Observation of a three-dimensional topological Dirac semimetal phase in high-mobility $\mathrm{Cd}_{3} \mathrm{As}_{2}$, Nat. Commun. 5, 3786 (2014).

[8] I. Crassee, R. Sankar, W.-L. Lee, A. Akrap, and M. Orlita, 3D Dirac semimetal $\mathrm{Cd}_{3} \mathrm{As}_{2}$ : A review of material properties, Phys. Rev. Mater. 2, 120302 (2018).

[9] T. Liang, Q. Gibson, M. N. Ali, M. Liu, R. J. Cava, and N. P. Ong, Ultrahigh mobility and giant magnetoresistance in the Dirac semimetal $\mathrm{Cd}_{3} \mathrm{As}_{2}$, Nat. Mater. 14, 280 (2015).

[10] T. Liang, J. Lin, Q. Gibson, T. Gao, M. Hirschberger, M. Liu, R. J. Cava, and N. P. Ong, Anomalous Nernst Effect in the Dirac Semimetal $\mathrm{Cd}_{3} \mathrm{As}_{2}$, Phys. Rev. Lett. 118, 136601 (2017).
[11] T. Schumann, L. Galletti, D. A. Kealhofer, H. Kim, M. Goyal, and S. Stemmer, Observation of the Quantum Hall Effect in Confined Films of the Three-Dimensional Dirac Semimetal $\mathrm{Cd}_{3} \mathrm{As}_{2}$, Phys. Rev. Lett. 120, 016801 (2018).

[12] H. T. Chorsi, S. Yue, P. P. Iyer, M. Goyal, T. Schumann, S. Stemmer, B. Liao, and J. A. Schuller, Widely tunable optical and thermal properties of Dirac semimetal $\mathrm{Cd}_{3} \mathrm{As}_{2}$, arXiv:1907.12105.

[13] B. Skinner and L. Fu, Large, nonsaturating thermopower in a quantizing magnetic field, Sci. Adv. 4, eaat2621 (2018).

[14] H. Wang, X. Luo, W. Chen, N. Wang, B. Lei, F. Meng, C. Shang, L. Ma, T. Wu, X. Dai et al., Magnetic-field enhanced high-thermoelectric performance in topological Dirac semimetal $\mathrm{Cd}_{3} \mathrm{As}_{2}$ crystal, Sci. Bull. 63, 411 (2018).

[15] T. Hosseini, N. Yavarishad, J. Alward, N. Kouklin, and M. Gajdardziska-Josifovska, Large thermopower, crystalline $\mathrm{Cd}_{3} \mathrm{As}_{2}$ by low-temperature vapor deposition for room temperature heat waste recovery, Adv. Electron. Mater. 2, 1500319 (2016).

[16] A. Pariari, N. Khan, R. Singha, B. Satpati, and P. Mandal, Tuning the scattering mechanism in the threedimensional Dirac semimetal $\mathrm{Cd}_{3} \mathrm{As}_{2}$, Phys. Rev. B 94, 165139 (2016).

[17] Z. Song, J. Zhao, Z. Fang, and X. Dai, Detecting the chiral magnetic effect by lattice dynamics in Weyl semimetals, Phys. Rev. B 94, 214306 (2016).

[18] P. Rinkel, P. L. S. Lopes, and I. Garate, Signatures of the Chiral Anomaly in Phonon Dynamics, Phys. Rev. Lett. 119, 107401 (2017).

[19] B. Cheng, T. Schumann, Y. Wang, X. Zhang, D. Barbalas, S. Stemmer, and N. Armitage, A large effective phonon magnetic moment in a Dirac semimetal, arXiv:1905.00309.

[20] D. P. Spitzer, G. A. Castellion, and G. Haacke, Anomalous thermal conductivity of $\mathrm{Cd}_{3} \mathrm{As}_{2}$ and the $\mathrm{Cd}_{3} \mathrm{As}_{2}-\mathrm{Zn}_{3} \mathrm{As}_{2}$ alloys, J. Appl. Phys. 37, 3795 (1966). 
[21] D. Armitage and H. Goldsmid, The thermal conductivity of cadmium arsenide, J. Phys. C 2, 2138 (1969).

[22] J. Zhu, T. Feng, S. Mills, P. Wang, X. Wu, L. Zhang, S. T. Pantelides, X. Du, and X. Wang, Record-low and anisotropic thermal conductivity of a quasi-one-dimensional bulk $\mathrm{ZrTe}_{5}$ single crystal, ACS Appl. Mater. Interfaces 10, 40740 (2018).

[23] S. Piscanec, M. Lazzeri, F. Mauri, A. C. Ferrari, and J. Robertson, Kohn Anomalies and Electron-Phonon Interactions in Graphite, Phys. Rev. Lett. 93, 185503 (2004).

[24] M. Lazzeri and F. Mauri, Nonadiabatic Kohn Anomaly in a Doped Graphene Monolayer, Phys. Rev. Lett. 97, 266407 (2006).

[25] T. Nguyen, F. Han, N. Andrejevic, P.-P. Ricardo, A. Apte, Y. Tsurimaki, Z. Ding, K. Zhang, A. Alatas, E. E. Alp, S. Chi, J. Fernandez-Baca, M. Matsuda, D. A. Tennant, Y. Zhao, Z. $\mathrm{Xu}$, J. W. Lynn, S. Huang, and M. Li, Discovery of topological singularity induced Kohn anomaly in Weyl semimetal, arXiv:1906.00539.

[26] G. Kresse and J. Furthmüller, Efficient iterative schemes for $a b$ initio total-energy calculations using a plane-wave basis set, Phys. Rev. B 54, 11169 (1996).

[27] G. Kresse and J. Furthmüller, Efficiency of ab-initio total energy calculations for metals and semiconductors using a planewave basis set, Comput. Mater. Sci. 6, 15 (1996).

[28] P. E. Blöchl, Projector augmented-wave method, Phys. Rev. B 50, 17953 (1994).

[29] G. Kresse and D. Joubert, From ultrasoft pseudopotentials to the projector augmented-wave method, Phys. Rev. B 59, 1758 (1999).

[30] J. P. Perdew, K. Burke, and M. Ernzerhof, Generalized Gradient Approximation Made Simple, Phys. Rev. Lett. 77, 3865 (1996).

[31] S. Steiner, S. Khmelevskyi, M. Marsmann, and G. Kresse, Calculation of the magnetic anisotropy with projected-augmentedwave methodology and the case study of disordered $\mathrm{Fe}_{1-x} \mathrm{Co}_{x}$ alloys, Phys. Rev. B 93, 224425 (2016).

[32] M. N. Ali, Q. Gibson, S. Jeon, B. B. Zhou, A. Yazdani, and R. J. Cava, The crystal and electronic structures of $\mathrm{Cd}_{3} \mathrm{As}_{2}$, the three-dimensional electronic analog of graphene, Inorg. Chem. 53, 4062 (2014).

[33] A. M. Conte, O. Pulci, and F. Bechstedt, Electronic and optical properties of topological semimetal $\mathrm{Cd}_{3} \mathrm{As}_{2}$, Sci. Rep. 7, 45500 (2017).

[34] K. Esfarjani, G. Chen, and H. T. Stokes, Heat transport in silicon from first-principles calculations, Phys. Rev. B 84, 085204 (2011).

[35] A. Togo and I. Tanaka, First principles phonon calculations in materials science, Scr. Mater. 108, 1 (2015).

[36] See Supplemental Material at http://link.aps.org/supplemental/ 10.1103/PhysRevResearch.1.033101 for more information.

[37] O. Hellman and D. A. Broido, Phonon thermal transport in $\mathrm{Bi}_{2} \mathrm{Te}_{3}$ from first principles, Phys. Rev. B 90, 134309 (2014).
[38] O. Delaire, J. Ma, K. Marty, A. F. May, M. A. McGuire, M.-H. Du, D. J. Singh, A. Podlesnyak, G. Ehlers, M. Lumsden et al., Giant anharmonic phonon scattering in PbTe, Nat. Mater. 10, 614 (2011).

[39] W. Kohn, Image of the Fermi Surface in the Vibration Spectrum of a Metal, Phys. Rev. Lett. 2, 393 (1959).

[40] G. Krizman, T. Schumann, S. Tchoumakov, B. A. Assaf, S. Stemmer, L. A. de Vaulchier, and Y. Guldner, Determination of the crystal field splitting energy in $\mathrm{Cd}_{3} \mathrm{As}_{2}$ using magnetooptics, Phys. Rev. B 100, 155205 (2019).

[41] L. Galletti, T. Schumann, O. F. Shoron, M. Goyal, D. A. Kealhofer, H. Kim, and S. Stemmer, Two-dimensional Dirac fermions in thin films of $\mathrm{Cd}_{3} \mathrm{As}_{2}$, Phys. Rev. B 97, 115132 (2018).

[42] S. Jandl, S. Desgreniers, C. Carlone, and M. Aubin, The Raman spectrum of $\mathrm{Cd}_{3} \mathrm{As}_{2}$, J. Raman Spectrosc. 15, 137 (1984).

[43] A. Sharafeev, V. Gnezdilov, R. Sankar, F. C. Chou, and P. Lemmens, Optical phonon dynamics and electronic fluctuations in the Dirac semimetal $\mathrm{Cd}_{3} \mathrm{As}_{2}$, Phys. Rev. B 95, 235148 (2017).

[44] J. Weszka, M. Renucci, and A. Zwick, Some aspects of Raman scattering in $\mathrm{Cd}_{3} \mathrm{As}_{2}$ single crystals, Phys. Status Solidi B 133, 57 (1986).

[45] S. Lee, K. Esfarjani, T. Luo, J. Zhou, Z. Tian, and G. Chen, Resonant bonding leads to low lattice thermal conductivity, Nat. Commun. 5, 3525 (2014).

[46] S.-Y. Yue, T. Xu, and B. Liao, Ultralow thermal conductivity in a two-dimensional material due to surface-enhanced resonant bonding, Mater. Today Phys. 7, 89 (2018).

[47] C. W. Li, O. Hellman, J. Ma, A. F. May, H. B. Cao, X. Chen, A. D. Christianson, G. Ehlers, D. J. Singh, B. C. Sales, and O. Delaire, Phonon Self-Energy and Origin of Anomalous Neutron Scattering Spectra in SnTe and PbTe Thermoelectrics, Phys. Rev. Lett. 112, 175501 (2014).

[48] C. W. Li, J. Hong, A. F. May, D. Bansal, S. Chi, T. Hong, G. Ehlers, and O. Delaire, Orbitally driven giant phonon anharmonicity in SnSe, Nat. Phys. 11, 1063 (2015).

[49] W. Li, J. Carrete, N. A. Katcho, and N. Mingo, ShengBTE: A solver of the Boltzmann transport equation for phonons, Comput. Phys. Commun. 185, 1747 (2014).

[50] J. E. Turney, E. S. Landry, A. J. H. McGaughey, and C. H. Amon, Predicting phonon properties and thermal conductivity from anharmonic lattice dynamics calculations and molecular dynamics simulations, Phys. Rev. B 79, 064301 (2009).

[51] W. Li, L. Lindsay, D. A. Broido, D. A. Stewart, and N. Mingo, Thermal conductivity of bulk and nanowire $\mathrm{Mg}_{2} \mathrm{Si}_{x} \mathrm{Sn}_{1-x}$ alloys from first principles, Phys. Rev. B 86, 174307 (2012).

[52] F. Giustino, Electron-phonon interactions from first principles, Rev. Mod. Phys. 89, 015003 (2017). 